\title{
Conjunctival Impression Cytology and Tear Function in Patients with Keratoconus
}

\author{
Deniz Ozarslan Ozcan, ${ }^{1}$ (1) Sait Coskun Ozcan, ${ }^{1}$ (i) Didar Gursoy ${ }^{2}$ \\ ${ }^{1}$ Department of Ophthalmology, Hatay Mustafa Kemal University Faculty of Medicine, Hatay, Turkey \\ ${ }^{2}$ Department of Pathology, Hatay Mustafa Kemal University Faculty of Medicine, Hatay, Turkey
}

\begin{abstract}
Objectives: The aim of this study was to evaluate the ocular surface alterations of conjunctival cytological changes and tear function in patients with keratoconus.

Methods: A total of 98 eyes with keratoconus and 68 eyes of age- and sex- matched healthy subjects were included in this prospective study. Ophthalmological evaluations, including Ocular Surface Disease Index (OSDI), tear break-up time (TBUT), ocular surface staining (OSS), Schirmer test, and conjunctival impression cytology (CIC), were performed.

Results: The mean participant age was $23.4 \pm 5.3$ years in the keratoconus group and $24.9 \pm 6.0$ years in the control group. In the keratoconus group, the mean OSDI and OSS scores were statistically significantly higher than those of the controls, and the Schirmer and TBUT results were statistically significantly lower (all $p<0.00 \mathrm{I}$ ). The severity of keratoconus was positively correlated with the CIC grade $(r=0.292 ; p=0.004)$.

Conclusion: Keratoconus results in deterioration of the ocular surface, including $\mathrm{CIC}$ and tear function parameters. The grade of keratoconus appears to be associated with conjunctival cytologic changes.

Keywords: Conjunctival impression cytology, keratoconus, ocular surface, tear functions
\end{abstract}

\section{Introduction}

Keratoconus is a progressive corneal ectasia that can lead to irregular astigmatism and loss of vision. The disease frequently begins during puberty; a reported incidence in the population is $I$ in 375 between the ages of $10-40$ years ( 1 ). Although the etiology of keratoconus remains unclear, a relationship between keratoconus and ocular surface disorders, such as allergies, corneal sensitivity, and tear film changes, has been reported in previous studies $(2,3)$.

Conjunctival impression cytology $(\mathrm{ClC})$ is a minimally invasive technique used to evaluate ocular surface cell mor- phology. It can help to identify a variety of ocular diseases, demonstrate the effects of treatments, and quantify inflammatory biomarkers $(4,5)$.

In clinical experience, ocular surface complaints are frequently seen in keratoconus patients in addition to visual symptoms. The hypothesis of this research was that diagnostic dry eye disease (DED) tests could reveal pathological changes on the ocular surface of keratoconus patients. This study is an analysis of DED tests used to evaluate conjunctival cytological changes and tear function in patients with keratoconus. The findings may help to explain the relationship between keratoconus and ocular surface alterations.

How to cite this article: Ozarslan Ozcan D, Ozcan SC, Gursoy D. Conjunctival Impression Cytology and Tear Function in Patients with Keratoconus. Beyoglu Eye J 2021; 6(2): 79-83.

Address for correspondence: Deniz Ozarslan Ozcan, MD. Hatay Mustafa Kemal Universitesi Tip Fakultesi, Goz Hastaliklari Anabilim Dali, Hatay, Turkey

Phone: +90 5052642015 E-mail: deniz.0609@hotmail.com

Submitted Date: January 20, 2021 Accepted Date: April 13, 2021 Available Online Date: June 08, 2021

${ }^{\circ}$ Copyright 2021 by Beyoglu Eye Training and Research Hospital - Available online at www.beyoglueye.com OPEN ACCESS This work is licensed under a Creative Commons Attribution-NonCommercial 4.0 International License. 


\section{Methods}

The study protocol was approved by the Hatay Mustafa Kemal University Tayfur Ata Sokmen Faculty of Medicine clinical research ethics committee (No: 2020/34, Date: 20/02/2020). The research was conducted in accordance with the ethical standards of Helsinki Declaration. All of the participants provided a written, informed consent form.

This prospective, case-controlled study was performed at the ophthalmology and pathology clinics of a university hospital. In all, 98 patients with keratoconus and 68 age- and sex- matched healthy subjects were enrolled.

The diagnosis of keratoconus was made based corneal thinning and ectasia findings detected in a clinical examination and corneal topography using a Sirius device (Costruzioni Strumenti Oftalmici SRL, Florence, Italy). The severity of keratoconus was determined according to the Amsler-Krumeich keratoconus classification (6). The control group consisted of participants who presented for a refraction examination and had 20/20 visual acuity. Only I eye of each participant was enrolled in the study. Patients with advanced keratoconus (grade 4) and those who had a history of ocular trauma or surgery, continuous use of ocular medication or contact lenses, or any ocular and systemic disorder that could affect the anterior surface of the eye were excluded from the study.

Ophthalmic evaluations, including the Ocular Surface Disease Index (OSDI; Allergan Inc., Irvine, CA, USA) questionnaire, tear break-up time (TBUT) measurement, ocular surface staining (OSS), the Schirmer I test, and $\mathrm{CIC}$ were performed for all of the study participants. The OSDI questionnaire was used to evaluate subjective levels of ocular discomfort and vision-related dysfunction in the previous 2 weeks (7). Subsequently, fluorescein was administered to the ocular surface and the patient was asked to blink naturally 3 times. TBUT was determined by measuring the interval between the instillation of topical fluorescein and the appearance of the first dry spots on the corneal tear film. Corneal and conjunctival staining were evaluated using fluorescein and Lissamine green dye (MilliporeSigma, Burlington,
MA, USA) Grading the severity of the OSS was performed according to a previously reported ocular staining grading system (8). The Schirmer I test was performed without anesthesia by measuring wetness 5 minutes after applying a paper strip $(5 \times 35 \mathrm{~mm})$ on the temporal one-third of the lower lid margin (9).

$\mathrm{CIC}$ was performed as follows: Following the administration of topical anesthesia with proparacaine, a cellulose acetate filter strip $(3 \times 5 \mathrm{~mm}$, pore size: $0.2 \mu \mathrm{m}$; Sartorius Stedim Biotech $\mathrm{GmbH}$, Göttingen, Germany) was placed on the temporal bulbar conjunctiva for 10 seconds. The strip was then fixed in $95 \%$ ethyl alcohol solution for 2 hours and subsequently stained with periodic acid-Schiff in the pathology department. All of the conjunctiva specimens collected were evaluated by a single pathologist (DG) who was unaware of the clinical details. Nelson's grading schema (grade 0-3) was used to categorize squamous metaplasia according to a microscopy evaluation $(10, \mathrm{II})$. The grade 0 and grade I nucleus/cytoplasm ratio is $1: 2$ to $1: 3$, and grade 2 and grade 3 have a ratio of $1: 4$ to $1: 6$.

\section{Statistical Analysis}

All of the statistical analyses were conducted using IBM SPSS Statistics for Windows, Version 21.0 software (IBM Corp., Armonk, NY, USA). Categorical variables were expressed as number and percentage, and quantitative variables were described using mean $\pm S D$. The normality of the distribution of the variables was assessed using the Kolmogorov-Smirnov test. According to the data distribution, the Student t-test or the Mann-Whitney $U$ test was used to determine significant differences between study groups. Correlations between the keratoconus stage and ocular surface parameters were analyzed with Spearman or Pearson correlation. The level of statistical significance was set at $p<0.05$.

\section{Results}

The demographic details of the study groups are summarized in Table I. In the keratoconus group, the mean age was $23.4 \pm 5.3$ years and $45.9 \%$ of the patients were female. The mean age was $24.9 \pm 6.0$ years and $44.1 \%$ of the participants

Table I. Demographic details of the study groups

\begin{tabular}{lccc} 
& Keratoconus patients $(\mathbf{n = 9 8})$ & Healthy controls $(\mathbf{n = 6 8 )}$ & p \\
\hline $\begin{array}{l}\text { Age (years) } \\
\text { Mean } \pm S D(\text { min-max })\end{array}$ & $23.4 \pm 5.3(16-37)$ & $24.9 \pm 6.0(17-44)$ & $0.092^{*}$ \\
Gender $(\mathrm{n})$ & & 30 & \\
Female & 45 & 38 & $0.819 * *$ \\
Male & 53 & & \\
\hline *: Student's t-test; **: Pearson chi-squared test. & & \\
\hline
\end{tabular}


were female in the control group. Table 2 illustrates the ocular surface parameters of the study groups. The OSDI, Schirmer test, TBUT, OSS scores, and CIC grades were clinically poorer in the keratoconus group compared with the healthy subjects. The mean OSDI $(35.49 \pm 12$.I vs $21.6 \pm 17.6)$ and OSS ( $1.94 \pm 1.3$ vs $1.02 \pm 0.9)$ scores were statistically significantly higher and the Schirmer $(4.32 \pm 2.6$ vs $12.56 \pm 3.8)$ and TBUT $(9.2 \pm 2.9$ vs $13.4 \pm 4.5)$ values were statistically significantly lower in the keratoconus group than those of the controls (all $\mathrm{p}<0.00 \mathrm{I}$ ). The $\mathrm{CIC}$ classification was grade 0 or I in $70 \%$ and grade 2 or 3 in $30 \%$ of the keratoconus group. In the control group, $91 \%$ were grade 0 or I and $9 \%$ were categorized as grade 2 or 3 .

Of the 98 keratoconic eyes, $42.9 \%$ eyes were classified as grade I keratoconus, $33.7 \%$ were grade 2 , and $22.4 \%$ were grade 3 . Analysis of the correlations between the keratoconus grade and ocular surface parameters revealed a significant positive correlation between the keratoconus grade and the $\mathrm{CIC}$ grade (Spearman correlation coefficient value [95\%]: 0.292; all $p=0.004$ ) (Table 3).

\section{Discussion}

In the present study, we investigated ocular surface alterations in patients with keratoconus and the relationship to the severity of the disease using diagnostic DED tests. The results of this research showed that patients with keratoconus had statistically significantly higher OSDI scores, reduced tear volumes, increased tear instability, greater ocular surface staining, and higher $\mathrm{CIC}$ grades than healthy controls. In addition, a statistically significantly positive correlation was observed between keratoconus and the $\mathrm{CIC}$ grade.

The degradation of corneal layers has a key role in the development of keratoconus (12). Collagen degradation products released into tears adversely affect the stability of the tear film (3). Furthermore, both stromal and epithelial thinning occur during the progression of keratoconus (13). Recent research has examined the role of inflammatory processes in the pathophysiology of keratoconus. Increased levels of inflammatory molecules, such as interleukin-6, tumor necrosis factor-alpha, and matrix metalloproteinase 9, have been demonstrated in keratoconus eyes in several studies

Table 2. Comparison of the ocular surface parameters of the study groups

\begin{tabular}{|c|c|c|c|}
\hline & $\begin{array}{l}\text { Keratoconus patients } \\
\qquad(n=98)\end{array}$ & $\begin{array}{l}\text { Healthy controls } \\
\qquad(n=68)\end{array}$ & $\mathbf{p}$ \\
\hline OSDI score (Mean \pm SD) & $35.49 \pm 12.1$ & $21.6 \pm 17.6$ & $<0.001 *$ \\
\hline Schirmer test $(\mathrm{mm})($ Mean $\pm \mathrm{SD})$ & $4.32 \pm 2.6$ & $12.56 \pm 3.8$ & $<0.001 * *$ \\
\hline TBUT (s) (Mean \pm SD) & $9.2 \pm 2.9$ & $13.4 \pm 4.5$ & $<0.001 * *$ \\
\hline OSS score (Mean $\pm S D)$ & $1.94 \pm 1.3$ & $1.02 \pm 0.9$ & $<0.001 * *$ \\
\hline CIC grade (n, \%) & & & $<0.001 * * *$ \\
\hline Grade 0 & $25(25.5)$ & $55(80.9)$ & \\
\hline Grade I & $44(44.9)$ & $7(10.3)$ & \\
\hline Grade 2 & $20(20.4)$ & $5(7.4)$ & \\
\hline Grade 3 & $9(9.2)$ & $\mathrm{I}(\mathrm{I} .5)$ & \\
\hline
\end{tabular}

Table 3. Correlation between keratoconus grade and ocular surface parameters

\begin{tabular}{|c|c|c|c|c|c|}
\hline & OSDI score & Schirmer test & TBUT & OSS score & CIC grade \\
\hline \multicolumn{6}{|c|}{ Keratoconus grade } \\
\hline$r$ & 0.085 & -0.049 & -0.112 & 0.107 & 0.292 \\
\hline $\mathrm{P}$ & $0.406 *$ & $0.586 * *$ & $0.27 I^{* *}$ & $0.296 * *$ & $0.004 * *$ \\
\hline $\mathrm{n}$ & 98 & 98 & 98 & 98 & 98 \\
\hline
\end{tabular}

*: Pearson's correlation analysis; **: Spearman's correlation analysis; CIC: Conjunctival impression cytology; OSDI: Ocular Surface Disease Index; OSS: Ocular surface staining;TBUT:Tear break-up time. 
$(14,15)$. Corneal trauma due to a contact lens or eye rubbing can provoke a strong inflammatory response in the corneal epithelium in patients with keratoconus $(16,17)$.

Higher OSDI and OSS scores, lower Schirmer I test results, and comparable TBUT measurements have been reported in keratoconus patients and healthy subjects (18). Moreover, patients with keratoconus have been observed to have a statistically significantly lower mucin cloud height and goblet cell layer thickness values and higher tear concentrations of di-adenosine tetraphosphate than healthy subjects. In our study, the TBUT measurements were statistically significantly lower in keratoconus patients, and the $\mathrm{ClC}$ findings were found to be positively correlated with the keratoconus grade using the Nelson grading schema. Other researchers also found lower TBUT measurements and higher OSS scores and $\mathrm{CIC}$ grades in patients with keratoconus compared with controls (3). As the keratoconus stage increased, the authors of that study found that the results of these measurements worsened. In contrast we only found a statistically significant correlation between the $\mathrm{ClC}$ grade and the keratoconus stage.

De Paiva et al. (19) reported that surface regularity indices, which are elevated in patients with DED, can be used to diagnose DED and predict the severity of the disease. Decreased TBUT measurements as a result of topographic steepening of the cornea in cases of keratoconus have been observed (3). However, another study evaluating the association between topographic/tomographic values and tear film parameters in keratoconus patients reported no interaction, as we also observed (20).

Our findings revealed significant levels of conjunctival squamous metaplasia and loss of goblet cells in the keratoconus eyes. There was a significant positive correlation between the keratoconus stage and the $\mathrm{CIC}$ grade. This may be related to the epithelial origin of the disease and the relationship between keratoconus and inflammation.

Limitations of the present study include the single-center design, a relatively small number of patients, and a lack of corneal sensitivity measurements and biomarkers to provide more evidence about tear components.

The results of the current study showed that the patients with keratoconus had clinically poorer diagnostic DED measurements than the healthy subjects. Our findings support the concept that adverse changes in the ocular surface in cases of keratoconus affect not only the cornea, but the tear film and conjunctival epithelium as well. In addition, the CIC grade was found to correlate with the stage of keratoconus. Therefore, $\mathrm{ClC}$ assessment may provide useful information to understanding the ocular surface alterations of patients with keratoconus. Our results should be confirmed by additional studies investigating the relationship between keratoconus and ocular surface parameters.

\section{Disclosures}

Ethics Committee Approval: Hatay Mustafa Kemal University Tayfur Ata Sokmen Faculty of Medicine Clinical Research Ethics Committee, protocol number: 2020/34, Date: 20/02/2020.

Peer-review: Externally peer-reviewed.

Conflict of Interest: None declared.

Authorship Contributions: Involved in design and conduct of the study (DOO, SCO, DG); preparation and review of the study (DOO, SCO); data collection (DOO, SCO, DG); and statistical analysis (DOO, SCO).

\section{References}

I. Godefrooji DA, de Wit GA, Uiterwaal CS, Imhof SM, Wisse RPL. Age specific incidence and prevalence of keratoconus: a nationwide registration study. Am J Ophthalmol 2017; I75:169_ 72. [CrossRef]

2. Sharma N, Rao K, Maharana PK, Vajpayee RB. Ocular allergy and keratoconus. Indian J Ophthalmol 20I3;6I:407-9. [CrossRef]

3. Dogru M, Karakaya $H$, Ozçetin $H$, Ertürk $H$, Yücel $A$, Ozmen $A$, et al. Tear function and ocular surface changes in keratoconus. Ophthalmology 2003; I 10: I I I0-8. [CrossRef]

4. Thatcher RW, Darougar S, Jones BR. Conjunctival impression cytology. Arch Ophthalmol 1977;95:678-81. [CrossRef]

5. Hagan S. Biomarkers of ocular surface disease using impression cytology. Biomark Med 20 I7; I : I I35-47. [CrossRef]

6. Krumeich $\mathrm{JH}$, Kezirian GM. Circular keratotomy to reduce astigmatism and improve vision in stage I and II keratoconus. J Refract Surg 2009;25:357-65. [CrossRef]

7. Schiffman RM, Christianson MD, Jacobsen G, Hirsch JD, Reis BL. Reliability and validity of the ocular surface disease index. Arch Ophthalmol 2000; I 18:615-2I. [CrossRef]

8. Whitcher JP, Shiboski CH, Shiboski SC, Heidenreich AM, Kitagawa $\mathrm{K}$, Zhang $\mathrm{S}$, et al. A simplified quantitative method for assessing keratoconjunctivitis sicca from the Sjögren's Syndrome International Registry. Am J Ophthalmol 2010;49:405-15. [CrossRef]

9. Wolffsohn JS, Arita R, Chalmers R, Djalilian A, Dogru M, Dumbleton K, et al. TFOS DEWS II Diagnostic Methodology report. Ocul Surf 2017;15:539-74. [CrossRef]

10. Nelson JD. Impression cytology. Cornea 1988;7:7I-8I. [CrossRef]

I I. Doughty MJ. Goblet cells of the normal human bulbar conjunctiva and their assessment by impression cytology sampling. Ocul Surf 2012;10:149-69. [CrossRef]

12. Kenney MC, Brown DJ, Rajeev B. Everett Kinsey lecture. The elusive causes of keratoconus: a working hypothesis. CLAO J 2000;26:10-3.

13. Reinstein DZ, Gobbe M, Archer TJ, Silverman RH, Coleman DJ. Epithelial, stromal, and total corneal thickness in keratoconus: three-dimensional display with artemis very-high frequency digital ultrasound. J Refract Surg 2010;26:259-7I. [CrossRef]

I4. Lema I, Duran JA. Inflammatory molecules in the tears of patients with keratoconus. Ophthalmology 2005; I I 2:654-9. [CrossRef] 
I5. Lema I, Sobrino T, Duran JA, Brea D, Diez-Feijoo E. Subclinical keratoconus and inflammatory molecules from tears. $\mathrm{Br} J \mathrm{Oph}$ thalmol 2009;93:820-4. [CrossRef]

16. Lema I, Duran JA, Ruiz C, Diez-Feijoo E, Acera A, Merayo J. Inflammatory response to contact lenses in patients with keratoconus compared with myopic subjects. Cornea 2008;27:75863. [CrossRef]

17. Balasubramanian SA, Pye DC, Wilcox MD. Effects of eye rubbbing on the levels of protease, protease activity and cytokines in tears: relevance in keratoconus. Clin Exp Optom 2013;96:2148. [CrossRef]
18. Carracedo G, Recchioni A, Alejandre-Alba N, Martin-Gil A, Crooke A, Morote IJ, et al. Signs and symptoms of dry eye in keratoconus patients: a pilot study. Curr Eye Res 2015;40:108894. [CrossRef]

19. De Paiva CS, Harris LD, Pflugfelder SC. Keratoconus-like topographic changes in keratoconjunctivitis sicca. Cornea 2003;22:22-4. [CrossRef]

20. Zemova, E, Eppig T, Seitz B, Toropygin S, Arnold S, Langenbucher $A$, et al. Interaction between topographic/tomographic parameters and dry eye disease in keratoconus patients. Curr Eye Res 2014;39:1-8. [CrossRef] 